\title{
A Functional Limit Theorem for Random Walk Conditioned to Stay Non-negative
}

\author{
A. Bryn-Jones \& R. A. Doney
}

First version: 3 October 2005

Research Report No. 14, 2005, Probability and Statistics Group School of Mathematics, The University of Manchester 


\title{
A functional limit theorem for random walk conditioned to stay non-negative.*
}

\author{
A. Bryn-Jones and R. A. Doney, Manchester University
}

\section{Introduction}

There is an increasing use in modelling of processes derived from random walks which are constrained to stay on a half-line; see [10] for a recent example of this. However the phrase "random walk conditioned to stay non-negative" has at least two different interpretations. In the first we consider the first $n$ values of a random walk conditioned on the event that all these values are non-negative; this is a discrete version of a meander, and it has been known for a long time that if the random walk is in the domain of attraction of a standard Normal law, a suitably scaled version of this process converges weakly to Brownian meander. (See [8], or [11].) The second interpretation involves conditioning on the event that the random walk never goes negative, and so can be thought of as a discrete version of the Bessel process. (Of course when the random walk oscillates, the conditioning event has zero probability, and we have to construct the conditioned process by the h-transform technique.) So a natural question, which we consider here, is whether there is an analogous weak convergence result for this version, the limit clearly being the Bessel process. Note that both the random walk meander and the Brownian meander can be represented as sections of the unconditioned process, which simplifies the proofs. In our case this device is not available and we fall back on the standard technique of establishing convergence of finite-dimensional distributions and tightness.

So let $S=\left\{S_{n}: n \geq 0\right\}$ denote a random walk on the integer lattice with starting state zero, that is $S_{0}=0$ and $S_{n}=\sum_{i=1}^{n} \xi_{i}$ where the $\xi_{i}$ are independent and identically distributed integer valued random variables. Additionally assume

*2000 MSC Subject Classification: 60J15, 60F15, 60K05, 60G40 
that $S$ is aperiodic and attracted to a standard Normal law, without centring, and let $a_{n}$ be the associated norming sequence. We define a normed and linearly interpolated version of $S$ by

$$
X_{n}(t)=\frac{S_{[n t]}}{a_{n}}+(n t-[n t]) \frac{\xi_{[n t]+1}}{a_{n}}, t \geq 0 .
$$

The weak convergence of the process $X_{n}=\left(X_{n}(t), t \geq 0\right)$ as $n \rightarrow \infty$ to a standard Brownian motion is Donsker's Invariance Principle (see for example Theorem 10.1 of [5]). Let $S^{*}$ denote " $S$ conditioned to stay non-negative" (in the sense of an h-transform) and $R_{n}=\left(R_{n}(t), t \geq 0\right)$ the corresponding linear interpolation of the normed version of $S^{*}$. The basic question we study here; is when does $R_{n}$ converge weakly as $n \rightarrow \infty$ to $R$, the 3 - dimensional Bessel process?

Actually, the fact that this happens in the special case that $S$ is simple symmetric random walk has been known for a long time, and indeed the classic paper on the Bessel process by Pitman [18] makes crucial use of this fact. On the other hand it is not obvious that the convergence of $R_{n}$ should hold under precisely the same conditions as that of $X_{n}$, and in fact the results in [15] seem to suggest that one might need to assume extra moment conditions. It turns out that this is not the case, and our main result, Theorem 2.1 below, shows that $X_{n}^{*}$ converges weakly to $R$ exactly when $S_{1}$ is in the domain of attraction of the standard Normal law. In the final section we also extend these results to the case that the initial value of $S$ is non-zero.

Actually our method of proof leads to a local limit theorem for $S_{n}^{*}$, and we formulate this as Theorem 2.2. Since $S^{*}$ is a h-transform of $S$ killed when it enters the negative half-line, which we denote by $\hat{S}$, we could also formulate Theorem 2.2 as a local limit theorem for $\hat{S}$. This in turn, by duality, gives the asymptotic behaviour of the joint mass function in the bivariate renewal process of descending ladder heights and times in $S$; in this form, which is stated as Proposition 1 below, it extends earlier results in Keener [16] and Alili and Doney [1] and [2].

\section{Conditioning $S$ to Stay Nonnegative}

Given a random walk $S=\left\{S_{n}: n \geq 0\right\}$, we denote $S$ conditioned to stay nonnegative by $S_{n}^{*}=\sum_{i=1}^{n} \xi_{i}^{*}$. In our integer-valued, oscillating case this is an honest Markov chain on $Z^{+}$, the non-negative integers, with transition probabilities

$$
P_{x}\left(S_{n}^{*}=y\right):=P\left(S_{n}^{*}=y \mid S_{0}^{*}=x\right)=\frac{V(y)}{V(x)} P_{x}\left(\widehat{S}_{n}=y\right), x, y \in Z^{+},
$$


where we have denoted by $\widehat{S}=\left\{\widehat{S}_{n}: n \geq 0\right\}$ the walk $S$ killed at time $T_{1}^{-}=$ $\min \left\{n: S_{n}<0\right\}$ and $V$ is the renewal function for the strict descending ladder height process of the walk $S$. ( See Bertoin and Doney [3]). The strict descending ladder variables for $S$ are defined as follows: Let $T_{k}^{-}$denote the $k^{\text {th }}$ strict descending ladder time, that is

$$
T_{k}^{-}=\min \left\{n>T_{k-1}^{-}: S_{n}<S_{T_{k-1}^{-}}\right\}
$$

for $k \geq 1$ where $T_{0}^{-} \equiv 0$. We let $H_{k}^{-}=-S_{T_{k}^{-}}$denote the $k^{\text {th }}$ descending ladder height so that the ladder process, $\left(T^{-}, H^{-}\right)=\left\{\left(T_{k}^{-}, H_{k}^{-}\right): k \geq 0\right\}$, is a bivariate renewal process. The renewal function associated with $H_{1}^{-}$is defined for $x \geq 0$ by

$$
V(x)=\sum_{k \geq 0} P\left(H_{k}^{-} \leq x\right),
$$

with the renewal mass function for the descending ladder time and height process given by

$$
v(n, x)=\sum_{k} P\left(T_{k}^{-}=n, H_{k}^{-}=x\right) .
$$

In a similar vein, the weak ascending ladder variables are denoted by $\left(T^{+}, H^{+}\right)=$ $\left\{\left(T_{k}^{+}, H_{k}^{+}\right): k \geq 0\right\}$ and are defined similarly, the $k^{\text {th }}$ weak ascending ladder time being

$$
T_{k}^{+}=\min \left\{n>T_{k-1}^{+}: S_{n} \geq S_{T_{k-1}^{+}}\right\}
$$

for $k \geq 1$, again letting $T_{0}^{+} \equiv 0$ and $H_{k}^{+}=S_{T_{k}^{+}}$. The renewal function associated with $H_{1}^{+}$is denoted throughout by $U(\cdot)$, and we define the renewal mass function for the ascending ladder time and height process to be

$$
u(n, x)=\sum_{k} P\left(T_{k}^{+}=n, H_{k}^{+}=x\right) .
$$

We can now formulate our main results.

Theorem 2.1. Let $S$ be an integer valued, aperiodic random walk which starts at 0 and is in the domain of attraction of a standard Normal law with norming sequence $a_{n}$. Then the process $R_{n}$ constructed from the conditioned random walk $S^{*}$ by

$$
R_{n}(t)=\frac{S_{[n t]}^{*}}{a_{n}}+(n t-[n t]) \frac{\xi_{[n t]+1}^{*}}{a_{n}}, t \geq 0,
$$

converges weakly as $n \rightarrow \infty$ to the 3-dimensional Bessel process $R$. 
Remark 1. Let

$$
A_{x}=\sum_{n \geq 0} \mathbf{1}_{\left\{S_{n}^{*} \leq x\right\}}, x>0,
$$

denote the total time that $S^{*}$ spends below the level $x$. Then a simple consequence of Theorem 2.1 is that as $x \rightarrow \infty$

$$
\frac{A_{x}}{a^{-1}(x)} \stackrel{d}{\rightarrow} \mathcal{A},
$$

where $a^{-1}$ is the inverse of a continuous interpolation of the norming sequence $a$, and $\mathcal{A}$ is the total time spent by the Bessel process below 1. This answers a question raised in [4].

The 3-dimensional Bessel process $R$ is the diffusion on the positive half line with transition density

$$
\begin{aligned}
P_{x}\left(R_{t} \in d y\right) & =\frac{1}{\sqrt{t}} r\left(\frac{x}{\sqrt{t}}, \frac{y}{\sqrt{t}}\right) d y, x>0 \\
\text { where } r(u, v) & =\sqrt{\frac{2}{\pi}} \frac{v}{u} \sinh (u v) \exp \left\{-\frac{u^{2}+v^{2}}{2}\right\},
\end{aligned}
$$

and entrance law with density

$$
\begin{aligned}
P_{0}\left(R_{t} \in d y\right) & =\frac{1}{\sqrt{t}} r_{0}\left(\frac{y}{\sqrt{t}}\right) d y \\
\text { where } r_{0}(v) & =\sqrt{\frac{2}{\pi}} v^{2} \exp \left\{-\frac{v^{2}}{2}\right\} .
\end{aligned}
$$

Theorem 2.2. Under the assumptions of Theorem 2.1, uniformly in $0 \leq z \leq$ $K a_{n}$, for any $K$,

$$
a_{n} P\left(S_{n}^{*}=z\right)=r_{0}\left(\frac{z}{a_{n}}\right)+o(1) \text { as } n \rightarrow \infty .
$$

We prove our main technical lemma in section 3, deduce Theorem 2.2 and Theorem 2.1 in section 4 , and in the final section we use a discrete version of Williams' path decomposition of the Bessel process to establish a version of Theorem 2.1 for a sequence of random walks which have the same step distribution but possibly different non-zero starting points. 


\section{A Bivariate Renewal Theorem}

Throughout this section we assume that $S$ is an integer-valued aperiodic random walk which starts at zero.

Proposition 1. Assume $S$ is attracted to a standard normal law with norming sequence $a_{n}$. Then we have

$$
P\left(\hat{S}_{n}=z\right)=u(n, z) \backsim n^{-1} U(z) P\left(S_{n}=z\right)
$$

uniformly as $n \rightarrow \infty$ for $0 \leq z \leq K a_{n}$ for any $K>0$.

Remark 2. We can deduce from this that, uniformly in $0 \leq z \leq K a_{n}$

$$
P\left(S_{m} \geq 0, m \leq n \mid S_{n}=z\right) \sim \frac{U(z)}{n} .
$$

This result was first proved in [16] under heavy assumptions on $S$ for a restricted range of values of $z$.

In preparation for the proof of Proposition 1 we state some known results concerning the ladder variables. The first is a version of the Wiener-Hopf factorisation taken from [1];

Lemma 1. Denote the first time at which the (weak, ascending) ladder height process exceeds the level $z$ by $N_{z}=\min \left\{k: H_{k}>z\right\}$; then for $k, z \in Z^{+}$

$$
\begin{array}{r}
P\left(T_{k}=n, H_{k}=z\right)=\frac{k}{n} P\left(H_{k-1} \leq S_{n}<H_{k}, S_{n}=z\right) \\
=\frac{k}{n} P\left(N_{z}=k, S_{n}=z\right),
\end{array}
$$

and

$$
n u(n, z)=E\left(N_{z}, S_{n}=z\right) .
$$

For the next result we write $\rho_{n}=P\left(S_{n} \geq 0\right)$, and define sequences $\left\{b_{n}\right\}$ and $\left\{c_{n}\right\}$ satisfying

$$
\log n=\sum_{m=1}^{\infty} \frac{1}{m} \rho_{m} \exp \left\{-\frac{m}{b_{n}}\right\} \text { and } c_{n}=a\left(b_{n}\right),
$$

where $a(\cdot)$ is any continuous increasing function such that $a(n)=a_{n}$. (That the definition of $\left\{b_{n}\right\}$ is legitimate is shown in [12].) Then, specialising results in [12] to the case $\rho=1 / 2$ gives; 
Lemma 2. When $S_{1}$ is attracted to a standard Normal law with norming sequence $a_{n}$, we have

$$
\left\{\left(\frac{T_{[k t]}}{b_{k}}, \frac{H_{[k t]}}{c_{k}}\right), t \geq 0\right\} \stackrel{d}{\rightarrow}\left\{\left(B_{t}, t\right), t \geq 0\right\}
$$

(ii) for any $\delta>0$,

$$
P\left((1-\delta) c_{m} \leq H_{m} \leq(1+\delta) c_{m} \mid T_{m}=s\right) \rightarrow 1
$$

as $m \rightarrow \infty$ uniformly for $d \leq \frac{s}{b_{m}} \leq D$ where $0<d<D<\infty$ are constants.

Proof of Proposition 1 The equality in (3.1) is a consequence of duality, and we also note that it suffices to prove that the asymptotic relation holds uniformly in the range

$$
\delta a_{n} \leq z \leq K a_{n}
$$

for any $K>1>\delta>0$. This is because a slight extension of Theorem 7 in [1], where it was assumed that $\operatorname{Var} S_{1}$ was finite, proves the result in the situation that $\frac{z}{a_{n}} \rightarrow 0$.

A consequence of the assumption that $S_{1}$ is in the domain of attraction of the Normal law is that both $H_{1}$ and $H_{1}^{-}$are relatively stable, and hence, using for example Theorem 8.8.1 of [6],

$$
U(z) \sim c^{-1}(z)=\frac{z}{l^{+}(z)}, V(z) \sim d^{-1}(z)=\frac{z}{l^{-}(z)}
$$

as $z \rightarrow \infty$, where $l^{ \pm}$are slowly varying functions, and $c^{-1}$ and $d^{-1}$ are inverses of the norming sequences for $H_{n}$ and $H_{n}^{-}$respectively. Using this in the representation

$$
n u(n, z)=E\left(N_{z}, S_{n}=z\right)
$$

from (3.2) we can write the asymptotic relation in (3.1) as

$$
\begin{aligned}
\sum_{k} k P\left(N_{z}=k, S_{n}=z\right) & \sim U(z) \sum_{k} P\left(N_{z}=k, S_{n}=z\right) \\
& \sim \frac{z}{l^{+}(z)} \sum_{k} P\left(N_{z}=k, S_{n}=z\right) .
\end{aligned}
$$


Given $\varepsilon>0$, we split these sums over the following ranges

$$
\begin{aligned}
& A_{1}: \quad k<m_{1}:=\left[(1-\varepsilon) \frac{z}{l^{+}(z)}\right], \\
& A_{2}: \quad m_{1} \leq k \leq m_{2}=\left[(1+\varepsilon) \frac{z}{l^{+}(z)}\right], \\
& A_{3}: \quad k>m_{2},
\end{aligned}
$$

and define for $i=1,2,3$

$$
\begin{aligned}
\Sigma_{i} & =\frac{l^{+}(z)}{z} \sum_{k \in A_{i}} k P\left(N_{z}=k, S_{n}=z\right) \\
P_{i} & =\sum_{k \in A_{i}} P\left(N_{z}=k, S_{n}=z\right),
\end{aligned}
$$

so that it suffices to prove that

$$
\Sigma_{1}+\Sigma_{2}+\Sigma_{3} \sim P_{1}+P_{2}+P_{3}=P\left(S_{n}=z\right) .
$$

Clearly

$$
(1-\varepsilon) P_{2} \leq \Sigma_{2} \leq(1+\varepsilon) P_{2}
$$

and since $P_{1} \geq \Sigma_{1}$ and $\Sigma_{3} \geq P_{3}$, the proof of the proposition lies in showing that $\Sigma_{3}$ and $P_{1}$ are both of order $o\left(P\left(S_{n}=z\right)\right)=o\left(\frac{1}{a_{n}}\right)$. (Here, and later, we use the standard bound

$$
c \leq a_{n} P\left(S_{n}=z\right) \leq d \text { for } 0 \leq z \leq K a_{n},
$$

without further comment. We will also write $c, d, .$. for finite positive constants whose values may change from line to line.)

By summation by parts

$$
\begin{aligned}
\Sigma_{3} & =\frac{l^{+}(z)}{z} \sum_{k \in A_{3}} k P\left(N_{z}=k, S_{n}=z\right) \\
& =m_{2} \frac{l^{+}(z)}{z} P\left(N_{z} \geq m_{2}, S_{n}=z\right)+\frac{l^{+}(z)}{z} \sum_{k>m_{2}} P\left(N_{z} \geq k, S_{n}=z\right) \\
& :=\Sigma_{3}^{(1)}+\Sigma_{3}^{(2)} .
\end{aligned}
$$


Note that from the definition of $m_{2}$

$$
\begin{aligned}
\Sigma_{3}^{(1)} & \leq c P\left(N_{z} \geq m_{2}, S_{n}=z\right) \\
& =c P\left(H_{m_{2}} \leq z, S_{n}=z\right) \\
& =c \sum_{x \leq z} \sum_{s \leq n} P\left(T_{m_{2}}=s, H_{m_{2}}=x\right) P\left(S_{n-s}=z-x\right) \\
& \leq c \sum_{s \leq n} P\left(T_{m_{2}}=s, H_{m_{2}} \leq z\right) \frac{1}{a_{n-s}} .
\end{aligned}
$$

Next take $\eta \in(0,1)$, and write

$$
\begin{aligned}
a_{n} \sum_{s \leq \eta n} P\left(T_{m_{2}}=s, H_{m_{2}} \leq z\right) \frac{1}{a_{n-s}} & \leq \frac{a_{n}}{a_{n(1-\eta)}} P\left(T_{m_{2}}<n \eta\right) \\
& =\frac{a_{n}}{a_{n(1-\eta)}} P\left(\frac{T_{m_{2}}}{b_{m_{2}}}<\frac{n \eta}{b_{m_{2}}}\right) .
\end{aligned}
$$

The bounds on $z$ in (3.6) and the fact that $c^{-1}(\cdot)=b^{-1}\left(a^{-1}(\cdot)\right)$ give

$$
b\left(m_{2}\right) \backsim b\left((1+\varepsilon) c^{-1}(z)\right) \backsim(1+\varepsilon)^{2} a^{-1}(z)
$$

and we conclude that for all sufficiently large $n$

$$
c n \leq b_{m_{2}} \leq d n,
$$

so that the last expression is asymptotically bounded by $\frac{1}{\sqrt{(1-\eta)}} G\left(\frac{\eta}{c}\right)$, where we have denoted by $G$ the stable $1 / 2$ distribution function. On the other hand, (3.9) 
allows us to use (3.5) of Lemma 2 to see that

$$
\begin{aligned}
& a_{n} \sum_{\eta n<s \leq n} P\left(T_{m_{2}}=s, H_{m_{2}} \leq z\right) \frac{1}{a_{n-s}} \\
= & \sum_{n \eta \leq s \leq n} P\left(H_{m_{2}} \leq z \mid T_{m_{2}}=s\right) P\left(T_{m_{2}}=s\right) \frac{a_{n}}{a_{n-s}} \\
= & o(1) \sum_{n \eta \leq s \leq n} P\left(T_{m_{2}}=s\right) \frac{a_{n}}{a_{n-s}} \\
= & o(1) \sum_{n \eta \leq s \leq n}\left(\frac{1}{b_{m_{2}}} g\left(\frac{s}{b_{m_{2}}}\right)+o\left(\frac{1}{b_{m_{2}}}\right)\right) \frac{a_{n}}{a_{n-s}} \\
\leq & o(1) \frac{1}{n} a_{n} \sum_{n \eta \leq s \leq n} \frac{1}{a_{n-s}} \\
\sim & o(1) \frac{1}{n} a_{n} \frac{n(1-\eta)}{a_{n(1-\eta)}} \sim o(1) \sqrt{(1-\eta)},
\end{aligned}
$$

in which $g$ denotes the density function for the stable $1 / 2$ distribution and we have used the local limit theorem and standard properties of regularly varying functions. As we are able to choose $\eta$ as small as we wish, $a_{n} \Sigma_{3}^{(1)}$ is $o(1)$, and it is left to demonstrate that $a_{n} \Sigma_{3}^{(2)}$ is also.

Note the following upper bound;

$$
\begin{aligned}
\Sigma_{3}^{(2)} & =\frac{l^{+}(z)}{z} \sum_{k \geq m_{2}} P\left(N_{z}>k, S_{n}=z\right) \\
& \leq c \frac{l^{+}(z)}{z} \sum_{s \leq n} \sum_{k \geq m_{2}} P\left(T_{k}=s, H_{k} \leq z\right) \frac{1}{a_{n-s}} d z \\
& \leq c\left(\sigma_{1}+\sigma_{2}\right)
\end{aligned}
$$

where for some $\eta>0$

$$
\begin{aligned}
\sigma_{1} & =\frac{l^{+}(z)}{z} \sum_{s<n \eta} \sum_{k \geq m_{2}} P\left(T_{k}=s, H_{k} \leq z\right) \frac{1}{a_{n-s}}, \\
\sigma_{2} & =\frac{l^{+}(z)}{z} \sum_{n \eta \leq s \leq n} \sum_{k \geq m_{2}} P\left(T_{k}=s, H_{k} \leq z\right) \frac{1}{a_{n-s}} .
\end{aligned}
$$


Denoting the renewal function for the increasing ladder times by $\Theta(\cdot)$, we have

$$
\begin{aligned}
a_{n} \sigma_{1} & \leq \frac{l^{+}(z)}{z} \sum_{k \geq m_{2}} P\left(T_{k}<n \eta, H_{k} \leq z\right) \frac{a_{n}}{a_{n(1-\eta)}} \\
& \leq \frac{l^{+}(z)}{z} \Theta(n \eta) \frac{a_{n}}{a_{n(1-\eta)}} .
\end{aligned}
$$

Since the ladder times are in the domain of attraction of a stable $1 / 2$ law with norming sequence $b$, the asymptotic behaviour of the function $\Theta(\cdot)$ is known (see for example page 361 of [6]) to be given by

$$
\Theta(n) \sim c b_{n}^{-1}
$$

The argument that established (3.9) shows that $\frac{z}{l^{+}(z)}$ is bounded above and below by constant multiples of the sequence $b_{n}^{-1}$ so

$$
\begin{aligned}
a_{n} \sigma_{1} & \leq \frac{c}{b_{n}^{-1}} \Theta(n \eta) \frac{a_{n}}{a_{n(1-\eta)}} \\
& \sim c \frac{b_{n \eta}^{-1}}{b_{n}^{-1} \sqrt{1-\eta}} \backsim \frac{c \sqrt{\eta}}{\sqrt{1-\eta}},
\end{aligned}
$$

and this can be made arbitrarily small by choice of $\eta$.

Next we take an arbitrary $\gamma$ and write $\sigma_{2}$ as the sum of the terms

$$
\begin{aligned}
\sigma_{2}^{(1)} & =\frac{l^{+}(z)}{z} \sum_{n \eta \leq s \leq n} \sum_{m_{2} \leq k \leq \gamma m_{2}} P\left(T_{k}=s, H_{k} \leq z\right) \frac{1}{a_{n-s}} \\
\sigma_{2}^{(2)} & =\frac{l^{+}(z)}{z} \sum_{n \eta \leq s \leq n} \sum_{k>\gamma m_{2}} P\left(T_{k}=s, H_{k} \leq z\right) \frac{1}{a_{n-s}} .
\end{aligned}
$$

Both these terms can bounded using similar arguments to the forgoing, but the details are omitted; this completes the proof that $a_{n} \Sigma_{3} \rightarrow 0$.

The final step in the proof is to show that $P_{1}$ is $o\left(\frac{1}{a_{n}}\right)$. The proof again relies on splitting the sum into several parts and showing that each is $o\left(\frac{1}{a_{n}}\right)$. We start by writing $P_{1}=P_{1}^{(1)}+P_{1}^{(2)}$, where

$$
\begin{aligned}
& P_{1}^{(1)}=P\left(H_{m_{1}}>z, T_{m_{1}} \leq n, S_{n}=z\right), \\
& P_{1}^{(2)}=P\left(H_{m_{1}}>z, T_{m_{1}}>n, S_{n}=z\right) .
\end{aligned}
$$


The first of these terms is shown to be sufficiently small by splitting it into two parts, the first being when the $m_{1}^{\text {th }}$ ladder time is close to zero and the second when it is bounded away from zero. Each part is then shown to be of the required order by a similar method to that used to bound $\sigma_{1}$; the details of this are also omitted. Letting $\nu_{n}=\max \left\{k: T_{k} \leq n\right\}$ denote the number of ladder epochs which occur by time $n$, we have

$$
P_{1}^{(2)}=P\left(S_{n}=z, \nu_{n}<m_{1}\right) .
$$

To bound this probability we again need to consider two separate cases depending on the value of $T_{\nu_{n}}$. Thus for $\delta \in(0,1)$ we first write

$$
\begin{aligned}
& P\left(T_{\nu_{n}}\right.\left.<n(1-\delta), H_{\nu_{n}} \geq z, S_{n}=z, \nu_{n}<m_{1}\right)= \\
& \sum_{\substack{w \geq z, k<m_{1} \\
l<n(1-\delta)}} P\left(T_{k}=l, H_{k}=w, v_{n}=k\right) P\left(S_{n-l}=z-w, \max _{j \leq n-l} S_{j}=0\right) .
\end{aligned}
$$

We can bound the final term in the above product by

$$
\begin{aligned}
& \sum_{y<0} P\left(S_{j} \leq 0 \text { for } j \leq \frac{(n-l)}{2}, S_{\frac{(n-l)}{2}}=y\right) P\left(S_{\frac{(n-l)}{2}}=z-\omega-y\right) \\
\leq & P\left(T_{1}^{-}>\frac{(n-l)}{2}\right) \frac{c}{a_{\frac{(n-l)}{2}}},
\end{aligned}
$$

and using this we see that the expression (3.10) is bounded above by a multiple of

$$
\begin{aligned}
& P\left(T_{\nu_{n}<n(1-\delta)}, H_{\nu_{n}} \geq z\right) P\left(T_{1}^{-}>\frac{n \delta}{2}\right) \frac{1}{a_{\frac{n \delta}{2}}} \\
\sim & \frac{1}{a_{n} \sqrt{\delta / 2}} P\left(T_{1}^{-}>\frac{n \delta}{2}\right)=o\left(\frac{1}{a_{n}}\right)
\end{aligned}
$$

as required. An obvious duality argument shows that

$$
P\left(T_{\nu_{n}} \geq n(1-\delta), S_{n} \in d z\right)=P\left(l_{n}<n \delta, S_{n} \in d z\right)
$$

where $l_{n}=\max \left\{k: S_{k} \leq 0\right\}$. Taking $\delta<\frac{1}{2}, l_{n}$ is no less than the number of non positive partial sums up until time $\left[\frac{n}{2}\right]$, which we will denote by $\Gamma_{n}$. The Arc Sine Theorem (see for example Feller [14], page 418) gives

$$
P\left(\Gamma_{n}<\frac{n \delta}{2}\right) \rightarrow \frac{2}{\pi} \arcsin \sqrt{\delta},
$$


and this in turn allows us the bound

$$
\begin{aligned}
P\left(T_{\nu_{n}} \geq n(1-\delta), S_{n}=z\right) & \leq a_{n} P\left(\Gamma_{n}<n \delta, S_{\left[\frac{n}{2}\right]}=y, S_{n}=z\right) \\
& \leq c P\left(\Gamma_{n}<n \delta\right) \rightarrow c \frac{2}{\pi} \arcsin \sqrt{2 \delta} .
\end{aligned}
$$

Since this can be made arbitrarily small by choice of $\delta$, we have $a_{n} P_{1} \rightarrow 0$, and the result is proved.

The proof of Theorem 2.2 also requires the following result:

Lemma 3. Uniformly in $0 \leq z \leq K a_{n}$ and $0 \leq y \leq K a_{n}$ for any $K$,

$$
\frac{U(z) V(y)}{n}=2 \frac{z}{a_{n}} \cdot \frac{y}{a_{n}}+o(1) \text { as } n \rightarrow \infty .
$$

Proof First we show that the result holds uniformly in the range

$$
\varepsilon a_{n} \leq z, y \leq K a_{n},
$$

for any $\varepsilon>0$. To see this, recall from (3.7) that we have

$$
U(x) \backsim \frac{x}{l^{+}(x)} \text { and } V(x) \backsim \frac{x}{l^{-}(x)} \text { as } x \rightarrow \infty,
$$

where $l^{+}$and $l^{-}$are slowly varying, and from this it follows that the characteristic functions $\psi^{ \pm}$of $H^{ \pm}$satisfy

$$
1-\psi^{ \pm}(s) \backsim \mp i s l^{ \pm}(1 / s) \text { as } s \downarrow 0 .
$$

From this and the Wiener-Hopf factorisation we get that, uniformly for $y \in[\varepsilon, K]$,

$$
\begin{aligned}
U\left(a_{n} z\right) V\left(a_{n} y\right) & \backsim \frac{a_{n} z}{l^{+}\left(a_{n}\right)} \cdot \frac{a_{n} y}{l^{-}\left(a_{n}\right)} \\
& \backsim \frac{z y}{1-\psi\left(1 / a_{n}\right)},
\end{aligned}
$$

where $\psi(s)=E\left(e^{i s S_{1}}\right)$. Since $S_{n} / a_{n}$ converges in distribution to the standard Normal law, a simple calculation gives

$$
n\left\{1-\psi\left(1 / a_{n}\right)\right\} \rightarrow \frac{1}{2},
$$


and this proves the result when (3.11) holds. But for $z \leq \varepsilon a_{n}$ we have

$$
\frac{U(z) V(y)}{n} \leq \frac{U\left(\varepsilon a_{n}\right) V\left(K a_{n}\right)}{n} \leq 3 K \varepsilon^{2} \text { for all large enough } n,
$$

and the result follows.

In the proof of Theorem 2.1 we need the following estimate for the transition function $P_{x}\left(\hat{S}_{n}=y\right)$.

Proposition 2. Suppose that $x_{n}$ and $y_{n}$ are integers such that

$$
\frac{x_{n}}{a_{n}} \rightarrow u>0, \frac{y_{n}}{a_{n}} \rightarrow v>0 \text { as } n \rightarrow \infty .
$$

Then

$$
a_{n} P_{x_{n}}\left(\hat{S}_{n}=y_{n}\right) \rightarrow \frac{u}{v} r(u, v) .
$$

Proof Writing $I_{n}=\min _{r \leq n} S_{r}$ and $\sigma_{n}=\min \left\{r \leq n: S_{r}=I_{n}\right\}$ we have the following identity;

$$
\begin{aligned}
P_{x_{n}}\left(\widehat{S}_{n}=y_{n}\right) & =\sum_{m=0}^{n-1} \sum_{\omega=0}^{x_{n} \wedge y_{n}} P_{x_{n}}\left(I_{n}=\omega, \sigma_{n}=m, S_{n}=y_{n}\right) \\
& =\sum_{m=0}^{n-1} \sum_{\omega=0}^{x_{n} \wedge y_{n}} v\left(m, x_{n}-\omega\right) u\left(n-m, y_{n}-\omega\right) .
\end{aligned}
$$

(A proof of this, which is a bivariate version of a result originally due to Spitzer [19], can be found in [2].) We show first that the tails in the $m$-summation in (3.13) can be ignored, viz

$$
\lim _{\eta \downarrow 0} \lim \sup _{n \rightarrow \infty} a_{n} \sum_{(m \wedge n-m) \leq n \eta} \sum_{\omega=0}^{x_{n} \wedge y_{n}} v\left(m, x_{n}-\omega\right) u\left(n-m, y_{n}-\omega\right)=0 .
$$

To see this, use (3.8) to see that for $\eta \leq 1 / 2$

$$
\begin{aligned}
& a_{n} \sum_{m \leq n \eta} \sum_{\omega=0}^{\infty} v\left(m, x_{n}-\omega\right) u\left(n-m, y_{n}-\omega\right) \\
= & a_{n} \sum_{m \leq n \eta} P_{x_{n}}\left(\sigma_{n}=m, S_{n}=y_{n}\right)=a_{n} P\left(\sigma_{n} \leq n \eta, S_{n}=y_{n}-x_{n}\right) \\
\leq & c P\left(\sigma_{n} \leq n \eta \mid S_{n}=y_{n}-x_{n}\right) \\
= & c P\left(\sigma\left(X_{n}(\cdot)\right) \leq \eta \mid X_{n}(1)=\left(y_{n}-x_{n}\right) / a_{n}\right) \\
\rightarrow & c P(\sigma(\tilde{B})) \leq \eta) .
\end{aligned}
$$


Here $\tilde{B}$ denotes a Brownian Bridge of unit length from 0 to $v-u$, which is known (see [17]) to be the weak limit of $X_{n}(\cdot)$ conditioned on $X_{n}(1)=\left(y_{n}-x_{n}\right) / a_{n}$, and $\sigma(\tilde{B})$ the time at which its minimum value occurs. Clearly $P(\sigma(\tilde{B})) \leq \eta) \rightarrow 0$ as $\eta \downarrow 0$, and since a similar argument applies to the other term, (3.14) follows. Next, arguments almost identical to those of Proposition 1 show that the function $v(\cdot, \cdot)$ has the asymptotic behaviour

$$
n v(n, x) \sim V(x-1) P\left(S_{n}=-x\right),
$$

as $n \rightarrow \infty$, uniformly for all $1 \leq x \leq a_{n} K$ where $K$ is any finite positive constant. Then applying Proposition 1 and (3.15) to (3.13), we see that (3.12) will follow from

$$
\begin{aligned}
& \lim _{\eta \downarrow 0} \lim _{n \rightarrow \infty} \sum_{n \eta<m<n(1-\eta)} \sum_{\omega=0}^{x_{n} \wedge y_{n}} \frac{V\left(x_{n}-\omega-1\right)}{m} \frac{U\left(y_{n}-\omega\right)}{n-m} \frac{a_{n}}{a_{m}} \frac{1}{a_{n-m}} \\
& \phi\left(\frac{x_{n}-\omega}{a_{m}}\right) \phi\left(\frac{y_{n}-\omega}{a_{n-m}}\right)=\frac{u}{v} r(u, v) .
\end{aligned}
$$

Using Lemma 3 and then making the substitutions $m=n \tau$ and $\omega=a_{n} w$ we see that this in turn will follow from

$$
2 \int_{0}^{u \wedge v} \int_{0}^{1} \frac{(u-w)(v-w)}{\tau^{\frac{3}{2}}(1-\tau)^{\frac{3}{2}}} \phi\left(\frac{u-w}{\tau}\right) \phi\left(\frac{v-w}{1-\tau}\right) d \tau d w=\frac{u}{v} r(u, v) .
$$

However since

$$
r(u, v) d v=\frac{v}{u} P_{u}\left(B_{1} \in d v, \inf _{s \leq 1} B_{s}>0\right)
$$

(3.16) can be rewritten as

$$
2 \int_{0}^{u \wedge v} \int_{0}^{1} g(u-w, \tau) g(v-w, 1-\tau) d \tau d w d v=P_{u}\left(B_{1} \in d v, \inf _{s \leq 1} B_{s}>0\right)
$$

where $B$ is a standard BM and $g$ is its first passage density, and this is immediate by decomposing the RHS according to the value of $\inf _{s \leq 1} B_{s}$ and using Lévy's factorisation at the maximum.

\section{Proofs}

\subsection{Proof of Theorem 2.2}

Since $P\left(S_{n}^{*}=z\right)=V(z) P\left(\hat{S}_{n}=z\right)$ Theorem 2.2 follows from Proposition 1, the Normal local limit theorem and the case $y=z$ of Lemma 2 . 


\subsection{Proof of Theorem 2.1}

The convergence of the finite-dimensional distributions follows easily from Theorem 2.2, Lemma 3 and Proposition 2, so we are left to prove tightness of the sequence $\left\{R_{n}\right\}$. Since $\left\{R_{n}\right\}$ is a linearly interpolated Markov Chain, according to Theorem 8.4 of [5], tightness will follow if we can show that for each positive $\varepsilon$ and $K$ there exist a $\lambda>1$ and an integer $n_{0}$ such that

$$
P\left(\max _{i \leq K n} S_{i}^{*} \geq \lambda a_{n}\right) \leq \frac{\varepsilon}{\lambda^{2}}
$$

holds for all $n \geq n_{0}$.

From the convergence of the marginal distribution of $R_{n}$ to that of $R$ and the explicit form of the latter, we can clearly choose $\lambda$ so that for all large $n$

$$
P\left(S_{[K n]}^{*} \geq \lambda a_{n}\right) \leq \frac{\varepsilon}{2 \lambda^{2}} ;
$$

thus (4.1) will hold if we can also arrange that

$$
P\left(\max _{i \leq K n} S_{i}^{*} \geq \lambda a_{n}, S_{[K n]}^{*}<\lambda a_{n}\right) \leq \frac{\varepsilon}{2 \lambda^{2}}
$$

To see this we argue that

$$
\begin{aligned}
& P\left(\max _{i \leq K n} S_{i}^{*} \geq \lambda a_{n}, S_{[K n]}^{*}<\lambda a_{n}\right) \\
= & \sum_{y<\lambda a_{n}} P\left(\max _{i \leq K n} \hat{S}_{i} \geq \lambda a_{n}, \hat{S}_{[K n]}=y\right) V(y) \\
\leq & P\left(\max _{i \leq K n} \widehat{S}_{i} \geq \lambda a_{n}, \widehat{S}_{[K n]}<\lambda a_{n}\right) V\left(\lambda a_{n}\right) \\
= & P\left(\max _{i \leq K n} S_{i} \geq \lambda a_{n}, \min _{i \leq K n} S_{i} \geq 0\right) V\left(\lambda a_{n}\right) \\
= & P\left(\max _{i \leq K n} S_{i} \geq \lambda a_{n} \mid T_{1}^{-}>K n\right) P\left(T_{1}^{-}>K n\right) V\left(\lambda a_{n}\right),
\end{aligned}
$$

and note that

$$
P\left(T_{1}^{-}>K n\right) V\left(\lambda a_{n}\right) \backsim \frac{\lambda}{\sqrt{K}} P\left(T_{1}^{-}>n\right) V\left(\lambda a_{n}\right) \rightarrow \frac{c \lambda}{\sqrt{K}} .
$$


On the other hand it is known from examples 1 and 3, Corollary 1 of [11] that

$$
P\left(\max _{i \leq n} S_{i} \geq \lambda a_{n} \mid T_{1}^{-}>n\right) \rightarrow P\left(\sup _{t \leq 1} B_{t}^{+} \geq \lambda\right),
$$

where $B^{+}=\left\{B_{t}^{+}: t \geq 0\right\}$ denotes standard Brownian Meander. The distribution of $\sup _{t \leq 1} B_{t}^{+}$is known explicitly (see [13]) and it is easy to check that the RHS of (4.3) is exponentially small in $\lambda$, so the bound (4.2) holds, and the sequence $\left\{R_{n}\right\}$ is tight.

\section{Starting away from zero}

In this section we consider briefly the situation where we 'condition to stay nonnegative' a sequence of random walks which each have the same step-distribution but possibly different starting points. In order to avoid cumbersome formulae, in this section we adopt the canonical notation and write $P_{x}$ for the measure of a random walk with initial value $x$, and $P_{x}^{*}$ for the measure of the corresponding conditioned version. This is specified by

$$
P_{x}^{*}\left(A \cap\left(S_{n}=y\right)\right)=\frac{V(y)}{V(x)} \hat{P}_{x}\left(A \cap\left(S_{n}=y\right)\right)
$$

for any event $A \in \mathcal{F}_{n}$, where $\hat{P}_{x}$ is the measure corresponding to $S$ killed at time $T_{1}^{-}=\min \left\{n: S_{n}<0\right\}$. For our purposes the following facts about this measure are useful.

Proposition 3. Write $\mu=\min \left(n: S_{n}=j\right)$ where $j=\inf _{n \geq 0}\left\{S_{n}\right\}$ is the all time minimum of $S$. Then

(i) under $P_{x}^{*}$ the post-minimum process $\left\{S_{n+\mu}-j, n \geq 0\right\}$ is independent of the pre-minimum process and has measure $P_{0}^{*}$;

(ii) the distribution of $j$ under $P_{x}^{*}$ is given by

$$
P(j=y)=\frac{v(x-y)}{V(x)}, 0 \leq y \leq x,
$$

where $v$ is the renewal mass function in the downgoing ladder height process.

This result is just a discrete-time version of a known result for Lévy processes in [9], and since the proof is similar we omit it. 
Remark 3. The cited result also contains a description of the pre-minimum process which can be taken over into our discrete setting, but we don't need this.

Now given a norming sequence $a_{k}$ and a sequence of integer starting points $i_{k}$ we define

$$
R_{k}(t)=\frac{S_{[k t]}}{a_{k}}+(k t-[k t]) \frac{\xi_{[k t]+1}}{a_{k}}, t \geq 0,
$$

so that under $P_{i_{k}}^{*}$ we have $R_{k}(0)=\frac{i_{k}}{a_{k}}:=z_{k}$.

Theorem 5.1. Suppose the assumptions of Theorem 2.1, hold and $z_{k} \rightarrow z \geq 0$ as $k \rightarrow \infty$. Then the process $R_{k}$ under $P_{i_{k}}^{*}$ converges weakly to the Bessel process with initial value $z$.

Proof For $z>0$ convergence of the finite-dimensional distributions follows from Proposition 2.2 and Lemma 2, so we need only establish tightness. But

$$
\begin{aligned}
& P_{i_{k}}^{*}\left(\max _{n \leq c k} S_{n} \geq \lambda a_{k}, S_{[c k]}<\lambda a_{k}\right) \\
\leq & P_{i_{k}}\left(\max _{n \leq c k} S_{n} \geq \lambda a_{k}, \min _{n \leq c k} S_{n} \geq 0, S_{[c k]}<\lambda a_{k}\right) \frac{V\left(\lambda a_{k}\right)}{V\left(i_{k}\right)} \\
\leq & P_{i_{k}}\left(\max _{n \leq c k} S_{n} \geq \lambda a_{k},\right) \frac{V\left(\lambda a_{k}\right)}{V\left(i_{k}\right)} \rightarrow P_{z}\left(\sup _{t \leq c} B_{t} \geq \lambda\right) \frac{\lambda}{z} .
\end{aligned}
$$

Since $P_{z}\left(\sup _{t \leq c} B_{t} \geq \lambda\right)$ is exponentially small in $\lambda$, the result follows as in the proof of Theorem 2.1. In the case $z=0$ this argument fails, and we need to use Proposition 3. This implies that it suffices to show that the pre-minimum process vanishes in probability as $k \rightarrow \infty$. The time at which $R_{k}$ first takes its all-time minimum value is $k^{-1} \mu$, and applying the Markov property at time $[k \varepsilon]$ and using 
(ii) of Theorem 5.1 gives

$$
\begin{aligned}
P_{i_{k}}^{*}(\mu & >k \varepsilon)=\sum_{x=0}^{i_{k}} \sum_{y \geq x} P_{i_{k}}^{*}\left(S_{[k \varepsilon]}=y, \min _{n \leq[k \varepsilon]} S_{n}=x, \min _{n>[k \varepsilon]} S_{n} \leq x\right) \\
& =\sum_{x=0}^{i_{k}} \sum_{y \geq x} \tilde{P}_{i_{k}}\left(S_{[k \varepsilon]}=y, \min _{n \leq[k \varepsilon]} S_{n}=x\right) \frac{V(y)}{V\left(i_{k}\right)} \frac{V(y)-V(y-x)}{V(y)} \\
& \leq \frac{1}{V\left(i_{k}\right)} \sum_{x=0}^{i_{k}} \sum_{y \geq x} P_{i_{k}}\left(S_{[k \varepsilon]}=y, \min _{n \leq[k \varepsilon]} S_{n}=x\right) V(x) \\
& \leq P_{i_{k}}\left(\min _{n \leq[k \varepsilon]} S_{n} \geq 0\right)=P_{0}\left(\min _{n \leq[k \varepsilon]} S_{n} \geq-a_{k} z_{k}\right) \rightarrow 0 .
\end{aligned}
$$

In this calculation we have used the fact that the renewal function $V$ is subadditive, and this also plays a rôle in showing that under $P_{i_{k}}^{*}$

$$
\sup _{t \leq k^{-1} \mu} R_{k}(t)=\frac{\max _{n \leq \mu} S_{n}}{a_{k}} \stackrel{P}{\rightarrow} 0 .
$$

We see this by applying the Markov property at $\tau_{k}:=\min \left\{n: S_{n} \geq \eta a_{k}\right\}$ to get, when $z_{k}<\eta$,

$$
\begin{aligned}
P_{i_{k}}^{*}\left(\max _{n \leq \mu} S_{n}\right. & \left.\geq \eta a_{k}\right)=\sum_{x=0}^{i_{k}} \sum_{y \geq \eta a_{k}} P_{i_{k}}^{*}\left(S_{\tau}=y, \min _{n \leq \tau} S_{n}=x, \min _{n>\tau} S_{n}>x\right) \\
& =\sum_{x=0}^{i_{k}} \sum_{y \geq \eta a_{k}} P_{i_{k}}^{*}\left(S_{\tau}=y, \min _{n \leq \tau} S_{n}=x\right) \frac{V(y)-V(y-x)}{V(y)} .
\end{aligned}
$$

Using the fact that

$$
\frac{V(y)-V(y-x)}{V(y)} \leq \frac{V(x)}{V(y)} \leq \frac{V\left(i_{k}\right)}{V\left(\eta a_{k}\right)} \rightarrow 0,
$$

since $i_{k} / a_{k} \rightarrow 0$, gives

$$
P_{i_{k}}^{*}\left(\max _{n \leq \mu} S_{n} \geq \eta a_{k}\right) \rightarrow 0,
$$

for each fixed $\eta>0$, and the result follows. 
Remark 4. Although we have considered only the case of random walks taking values on the integer lattice, it is not difficult to extend our results to the nonlattice case; for details see [7]. On the other hand, extending the results to random walks in the domain of attraction of stable laws seems quite challenging, if only because we have little explicit knowledge of "stable processes conditioned to stay non-negative".

\section{References}

[1] Alili, L. and Doney, R.A. (1997). Wiener-Hopf factorization revisited and some applications. Stoch. Stoch. Reports 66, 87-102.

[2] Alili, L. and Doney, R.A. (2001). Martin boundaries associated with a killed random walk. Ann. I. H. Poincaré 37, 313-338.

[3] Bertoin, J. and Doney, R.A. (1994). On conditioning a random walk to stay nonnegative, Ann. Probab. 22, 2152-2167.

[4] Biggins, J. D. (2003). Random walk conditioned to stay positive. J. London Math. Soc. 67, 259-272.

[5] Billingsley, P. (1968). Convergence of Probability Measures. Wiley, New York and London.

[6] Bingham, N.H., Goldie, C.M and Teugels, J.L. (1987). Regular Variation. Cambridge University Press.

[7] Bryn-Jones, A. (2003). Ph. D. thesis, University of Manchester.

[8] Bolthausen, E. (1972). On a functional central limit theorem for random walk conditioned to stay positive. Ann. Probab. 4, 480-485.

[9] Chaumont, L. (1996). Conditionings and path decompositions for Lévy processes. Stoch. Processes Appl. 64, 39-54.

[10] Caravenna, F., Giacomin, G. and Zambotti, L. (2005). A renewal theory approach to periodic copolymers with adsorption. Preprint.

[11] Doney, R.A. (1985). Conditional limit theorems for asymptotically stable random walks. Z. Wahrscheinlichkeitsth. 70, 351-360. 
[12] Doney, R.A. and Greenwood, P.E. (1993). On the joint distribution of ladder variables of random walk. Probab. Theory Relat. Fields. 94, 457-472.

[13] Durrett, R.T., Iglehart, D.L. and Miller, D.R. (1977). Weak convergence to Brownian meander and Brownian excursion. Ann. Probab. 5(1), 117-129.

[14] Feller, W. (1971). An Introduction to Probability Theory and Its Applications. 2nd Ed. Wiley, New York, London.

[15] Hambly, B. M., Kersting, G. and Kyprianou, A. E. (2003). Law of the iterated logarithm for oscillating random walks conditioned to stay non-negatuive. Stoch. Proc. Appl. 108, 327-343.

[16] Keener, R. W. (1992). Limit theorems for random walk conditioned to stay positive. Ann. Probab. 20, 801-824.

[17] Liggett, T. M. (1968). An invariance principle for conditioned sums of independent random variables. J. Math. Mech., 18, 412-422.

[18] Pitman, J. (1975). One-dimensional Brownian motion and the threedimensional Bessel process. Adv. Appl. Probab. 7, 511-526.

[19] Spitzer, F. (1964). Principles of Random Walk. D. Van Nostrand Company Inc., New Jersey. 


\begin{abstract}
In this paper we consider an aperiodic integer-valued random walk $S$ and a process $S^{*}$ which is an harmonic transform of $S$ killed when it first enters the negative half; informally $S^{*}$ is "S conditioned to stay non-negative". If $S$ is in the domain of attraction of the standard Normal law, without centring, a suitably normed and linearly interpolated version of $S$ converges weakly to standard Brownian motion, and our main result is that under the same assumptions a corresponding statement holds for $S^{*}$, the limit of course being the 3-dimensional Bessel process. Since this process can be thought of as Brownian motion conditioned to stay non-negative, in essence we our result shows that the interchange of the two limit operations is valid.

We also establish some related results, including a local limit theorem for $S^{*}$, and a bivariate renewal theorem for the ladder time and height process, which may be of independent interest.
\end{abstract}

